\title{
Effect of antiresorptive drugs in the alveolar bone healing. A histometric and immunohistochemical study in ovariectomized rats
}

\author{
Gabriel Ramalho-Ferreira ${ }^{1}$. Leonardo Perez Faverani ${ }^{1}$. \\ Gustavo Antonio Correa Momesso ${ }^{1}$ - Eloá Rodrigues Luvizuto ${ }^{1}$. \\ Igor de Oliveira Puttini ${ }^{1} \cdot$ Roberta Okamoto $^{1,2}$
}

Received: 20 December 2015 / Accepted: 7 July 2016 / Published online: 27 July 2016

(C) Springer-Verlag Berlin Heidelberg 2016

\begin{abstract}
Objectives The aim of this study is to evaluate the alendronate and raloxifene influence in the alveolar healing process of osteoporotic rats.

Materials and methods Sixty-four female rats were divided in four groups: sham rats (SHAM), ovariectomized rats and no medical treatment (OVX NT), ovariectomized rats and submitted to alendronate treatment (OVX ALE), and ovariectomized and submitted to raloxifene treatment (OVX RAL). The histomorphometrical and immunohistochemical analysis was performed. The quantitative data were analyzed through Kruskal-Wallis and Dunn tests $(\alpha=0.05)$.

Results In the longest period, SHAM and OVX RAL groups showed the better bone formation responses $(P<0.05)$. The worst bone formation response was observed in the group OVX NT. OVX RAL group showed the better response at 42 days. OVX ALE group showed a favorable response at 14 days, in comparison with OVX RAL group, but a reduced response at 42 days. It was possible to observe a mature bone in SHAM group at 14 days and an immature bone in the OVX NT group. An intermediate quality bone was observed in the groups OVX ALE and OVX RAL.
\end{abstract}

Roberta Okamoto is affiliated with Research productivity scholarship (Process:308126/2014-9).

Gustavo Antonio Correa Momesso gustavomomesso@gmail.com

1 Department of Surgery and Integrated Clinic, Division of Oral and Maxillofacial Surgery, Aracatuba Dental School, Universidade Estadual Paulista (UNESP), Rua José Bonifácio, 1193, CEP 16015-050, Araçatuba, São Paulo, Brazil

2 Department of Basic Sciences, Aracatuba Dental School, Universidade Estadual Paulista (UNESP), São Paulo, Brazil
Conclusion Alendronate and raloxifene treatment improved the alveolar healing process in osteoporotic rats, but not enough to achieve the histometrical and protein expression values that were observed in the SHAM group.

Clinical relevance Alendronate is largely used as a potent antiresorptive agent. Otherwise, considering the undesirable effects in relation to the alveolar healing, other antiosteoporosis medications should be studied. Raloxifene seems to be a good candidate once its action mechanism involves the activation of osteoblasts.

Keywords Alendronate $\cdot$ Raloxifene $\cdot$ Osteocalcin . Osteoprotegerin $\cdot$ RANKL

\section{Introduction}

Menopause-related estrogen (E2) deficiency causes osteopenia in approximately two thirds of women. On the other hand, age-related estrogen deficiency causes osteoporosis $[1,2]$. The process of bone loss with aging differs from that induced by a decrease in gonadal function. In the former process, a decrease of intestinal calcium absorption from diet results in bone loss, especially in the cortical bone [3, 4]. In the latter process, a reduction of sexual female hormone levels produces an increase in the number and activity of osteoblasts and osteoclasts, which in turn produces an imbalance between bone formation (inadequate osteogenesis) and resorption (excessive osteoclastogenesis), resulting in bone loss, especially in the trabecular bone [5]. An increase in the number of osteoclast precursors is thought to occur as a consequence of increased interleukin (IL)-6 production by preosteoblasts.

Bisphosphonates are antiresorptive drugs that were developed to treat skeletal diseases, such as multiple myeloma and 
bone metastasis, and to prevent fractures in patients with osteoporosis $[6,7]$. To prevent fractures, alendronate sodium (4-amino-1-hydroxybutylidene), a second-generation bisphosphonate, is most indicated because it has less side effects than first-generation bisphosphonates. These drugs are synthetic analogs of the endogenous pyrophosphate, which exerts its antiresorptive action through binding with hydroxyapatite, thus inhibiting osteoclast development and migration while promoting osteoclast apoptosis [8]. Because of its great affinity to the bone matrix, approximately $50 \%$ of the absorbed bisphosphonate dose remains associated with the bone and the remainder is slowly eliminated, with a half-life of approximately 10 years and no signs of metabolization [9].

Selective estrogen receptor modulators (SERMs) are a class of drugs that act as estrogen receptor agonists in some tissues and antagonists in others. These medications are used to treat or prevent a range of hormone-dependent conditions, such as breast cancer, osteoporosis, and cardiovascular disease [10]. Raloxifene, a benzothiophene analog, is the only SERM approved by the Food and Drug Administration (FDA) to treat and prevent osteoporosis in the USA and in several other countries based on a reduction in vertebral fracture incidence in women with postmenopausal osteoporosis [11]. Raloxifene has also been shown to prevent cardiovascular disease in highrisk individuals by regulating circulating lipid levels [12]. The effects of raloxifene in the endometrium are minimal.

Ovariectomy has been a useful model to investigate the relationship between estrogen deficiency and bone metabolism. However, some studies have shown that ovariectomyinduced estrogen deficiency alone is not enough to induce maxillary osteoporosis in female rats over a period of 11 weeks. On the other hand, ovariectomy combined with a low-calcium diet decreases bone mass in the maxilla twice as much as in the proximal tibial metaphysis after a 5-week period $[3,5]$.

Alveolar healing - a process during which newly produced bone completely fills the tooth socket that was previously occupied by the tooth root-is an interesting model to study bone tissue dynamics [13,14]. An important question consists in, "How to produce mineralized tissue with adequate quality to support the mechanical stress produced by implantsupported prostheses?" $[15,16]$.

Several questions must be answered in order to achieve better bone quality. Considering the increase in life expectation, the proportion of the osteoporotic population that require dental treatment to replace missing teeth, and the need to better understand the peculiarities of alveolar healing, the present study aimed to evaluate the influence of alendronate and raloxifene during the alveolar healing process in female rats with induced osteoporosis.

\section{Material and methods}

Sixty-four female adult (3 months old) Wistar rats (Rattus norvegicus albinus) were divided into four groups. The rats were kept in cages and were feed with a balanced diet (NUVILAB, Curitiba, PR, Brazil) containing $1.4 \% \mathrm{Ca}$ and $0.8 \% \mathrm{P}$ and watered ad libitum.

After sham and ovariectomy surgeries, the rats of the sham group with balanced diet continue receiving the same diet (NUVILAB) containing $1.4 \% \mathrm{Ca}$ and $0.8 \% \mathrm{P}$ and water ad libitum, while the rats of the ovariectomy group received a poor-calcium diet containing $0.1 \% \mathrm{Ca}$ and $0.5 \% \mathrm{P}$ (RHOSTER Ind. Com., Vargem Grande Paulista, SP, Brazil) and water ad libitum. Evidence of osteoporosis installation was confirmed by results of microtomography performed in our group. It was observed decreasing of bone mineral density in ovariectomized tibias rats when compared to sham rats [17].

The experimental groups were the following: sham rats fed a normal diet (SHAM), ovariectomized rats given a lowcalcium diet but no drug treatment (OVX NT), ovariectomized rats given a low-calcium diet and treated with alendronate (OVX ALE), and ovariectomized rats given a low-calcium diet and treated with raloxifene (OVX RAL).

The groups were divided into two subgroups according to follow-up of alveolar healing: rats submitted to right upper incisive extraction and evaluation of alveolar healing at 14 days after the surgery (subgroup 14), and rats submitted to right upper incisive extraction and evaluation of alveolar healing at 42 days after the surgery (subgroup 42).

This study was approved by the Ethics Committee in Animal Experimentation (approval \#2010/003045).

Initially, the rats were kept in cages for the daily study of the estrous cycle as described by Long and Evans [18]. Briefly, 1-2 drops of saline solution were introduced intravaginally, aspired, and placed on a microscope slide for observation. After achieving two or three regular cycles, the animals were selected for the experiment.

Rats of the OVX NT, OVX ALE, and OVX RAL groups were anesthetized with Coopazine ${ }^{\circledR}(10 \mathrm{mg} / \mathrm{kg}$ xylazyne; Coopers Ltd., Brazil) and Vetaset ${ }^{\circ}(75 \mathrm{mg} / \mathrm{kg}$ ketamine hydrochloride; Saúde Animal Ltd., Fort Dodge, IA, USA) and submitted to bilateral ovariectomy using a dorsal approach. In the sham group, the ovaries were exposed but not excised.

Eight days after the ovariectomy, rats received $0.1 \mathrm{mg} / \mathrm{kg} /$ day alendronate for 30 days via gavage [19] or $1 \mathrm{mg} / \mathrm{kg} / \mathrm{day}$ raloxifene for 30 days via gavage [20]. The medication was administered until the end of the experiment (i.e., euthanasia) for a total of 44 and 72 days, respectively.

Thirty days after the beginning of treatment with alendronate or raloxifene, rats were anesthetized via intramuscular infiltration of Coopazine ${ }^{\circledR}(10 \mathrm{mg} / \mathrm{kg}$ xylazyne; Coopers Ltd.) and Vetaset ${ }^{\circledR}(75 \mathrm{mg} / \mathrm{kg}$ ketamine hydrochloride; Saúde Animal Ltd.) according to the manufacturer's indications. The surgical 
area was scrubbed with povidone iodine topical solution (Riodeine Indústria Química e Farmacêutica Rio Química, Ltd.), and the right upper incisive was extracted using a specially adapted instrument [13]. The gingival mucosa was sutured with polyglactin 910 (Vicryl 4.0; Johnson \& Johnson, São José dos Campos, São Paulo, Brazil).

Euthanasia was performed at 14 and 42 days after tooth extraction under an anesthetic overdose. The right maxilla was excised for histological evaluation of paraffin slices of the dental sockets.

The specimens were fixed in formol, decalcified with EDTA $(18 \%)$, and dehydrated using a graded alcohol series. Next, diaphonization was performed in xylol. Finally, the specimens were embedded in paraffin and cut into 5- $\mu \mathrm{m}$-thick sections.

Slides were used for hematoxylin and eosin (HE) staining or immunohistochemical reactions. A single, blinded investigator performed all the analyses.

HE-stained slides were used for histometric analysis. The dental socket was divided into three parts: cervical, medium, and apical. The events were observed in the medium third. The histometric analysis was performed using point counting and planimetry methods. The dental socket slides were first photographed using a digital camera system with a $\times 10$ objective adapted to a Nikon microscope (Eclipse 80i; Shinagawa, Tokyo, Japan) and processed with Image Pro software (Plus 5.1; Bethesda, MD, USA). To estimate the volume of newly formed bone tissue, two Merz grids were superimposed in each medium third of the dental socket using Adobe Photoshop (version 6.0). Each grid contained 100 equidistant points; thus, the two grids contained a total of 200 points.

Data on blood clot area, connective tissue, and bone tissue (\% of total area) were statistically evaluated by Kruskal-Wallis test and Nemenyi-Damico-Wolfe-Dunn test.

For immunohistochemistry, endogenous peroxidase was inhibited with inhibition hydrogen peroxide. Next, slides were subjected to an antigen recovery protocol with citrate phosphate buffer ( $\mathrm{pH}$ 6.0). The primary antibodies used were polyclonal goat antibodies against tartrate-resistant acid phosphatase (TRAP; SC 30832), osteoprotegerin (OPG; SC 21038), receptor activator of nuclear factor $\mathrm{KB}$ ligand (RANKL; SC 7627), and osteocalcin (SC 18319) (Santa Cruz Biotechnology). These antibodies were used to evaluate the cellular responses related to bone resorption (TRAP), remodeling (OPG and RANKL), and mineralization (osteocalcin). The secondary antibody was a biotinylated rabbit anti-goat antibody (Pierce Biotechnology). Signal amplification was achieved using avidin and biotin (Vector Laboratories) and diaminobenzidine (Dako) was the chromogen. At the end of the diaminobenzidine revelation of the reaction, the sections were counterstained with Harris hematoxylin.

Manual counting of labeled cells in predefined areas involved in bone tissue dynamics was performed using a Nikon microscope (Eclipse 80 i) with a $\times 20$ objective. Negative controls were used to test antibody specificity. Labeling intensity was classified as light $(++)$, moderate $(+++)$, and intense $(++++)$. The evaluation was performed across the entire dental socket extension at 14 or 42 days after tooth extraction.

\section{Results}

\section{Clinical and microscopy analysis}

Changes in the estrous cycle were observed in response to ovariectomy, as expected. The OVX NT, OVX ALE, and OVX RAL groups were in the diestrus stage of the cycle, which is characterized by the predominance of leukocytes. Sham rats were in the diestrus, proestrus, estrus, or metaestrus stage of the cycle.

To characterize the dynamics of the alveolar healing process, blood clot, connective tissue, and bone tissue formation were analyzed quantitatively in all groups (Fig. 1). During the course of the process, blood clot and connective tissue area decreased and bone tissue increased in the interior of the dental socket.
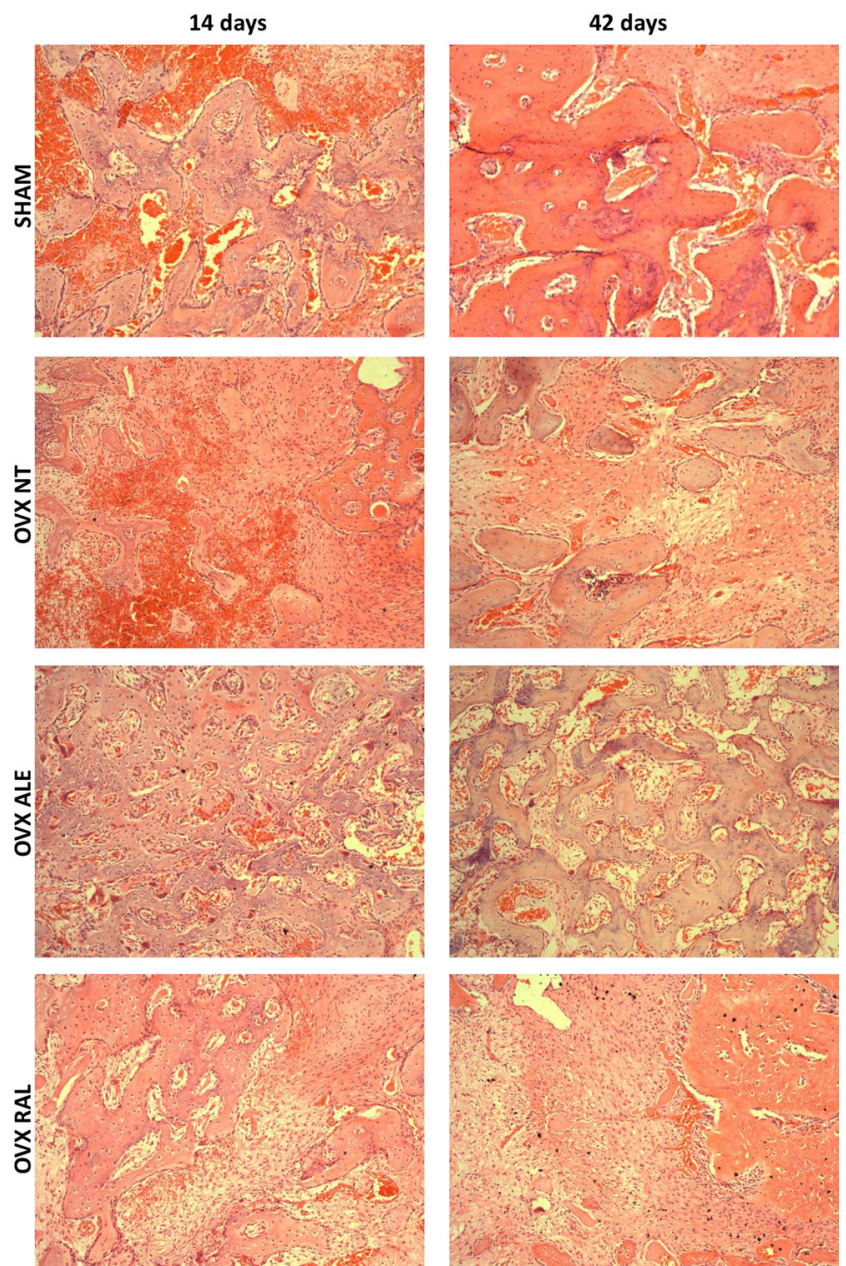

Fig. 1 Histological slices stained with hematoxylin and eosin (HE) in the cervical third of the dental socket. Sham (14 and 42 days), OVX NT (14 and 42 days), OVX ALE (14 and 42 days), and OVX RAL (14 and 42 days). Original, $\times 100$ 
This observation was common to all four experimental groups (SHAM, OVX NT, OVX ALE, and OVX RAL).

At 14 days after the surgery, the best repair responses were observed in the SHAM and the OVX ALE groups. The highest delay was observed in the OVX NT group. Alendronate and raloxifene treatment improved bone formation in rats with induced osteoporosis.

At 42 days after the surgery, the OVX RAL group had a moderately superior response compared to the SHAM group. The OVX ALE group had a better response than the OVX RAL group at 14 days, but still inferior to that of the SHAM group (Fig. 1).

\section{Histometric analysis}

At 14 days after the surgery, the OVX RAL group had the smallest, and the OVX NT group had the largest blood clot area; there was a statistically significant difference between OVX RAL and OVX NT $(P<0.05)$ (Fig. 2). The OVX ALE group had the smallest, and the OVX RAL group had the largest connective tissue area; there was a statistically significant difference between OVX RAL and OVX ALE, and between SHAM and OVX ALE $(P<0.05)$ (Fig. 3). The OVX NT group had the smallest, and the OVX ALE group had the largest bone tissue area; however, there were no significant differences between the groups.

At 42 days after the surgery, the SHAM group had the smallest, and the OVX ALE group had the largest blood clot area; there was a statistical significant difference between OVX RAL and SHAM $(P<0.05)$ (Fig. 4). The OVX RAL group had the smallest, and the OVX NT group had the largest connective tissue area; however, there were no significant differences between the groups. The OVX NT group had the smallest, and the OVX RAL group had the largest bone tissue area; however, there were no significant differences between the groups.

\section{Immunohistochemical analysis}

Immunohistochemical reactions were performed to evaluate the cellular responses through different synthesized proteins during the alveolar healing process at 14 days after extraction surgery (the time point of greatest expression) and at 42 days (for long-term evaluation of bone healing).

\section{OPG labeling}

In the SHAM group, OPG was detected in the cells of osteoblastic lineage, specifically in osteocytes (Fig. 5). OPG was also detected in the extracellular matrix of connective tissue and on the tissue precursor of bone trabeculae. OPG was moderately expressed at 14 days (Fig. 5) but only lightly expressed at 42 days (Fig. 5).

In the OVX NT group, OPG was detected in the extracellular matrix as well as in the connective tissue, but not in the bone trabeculae. OPG was lightly expressed at 14 days (Fig. 6) and at 42 days.

In the OVX ALE group, OPG was detected in the extracellular matrix, connective tissue, and discretely in the forming trabecular bone. OPG was moderately expressed at 14 days (Fig. 7), but the intensity decreased at 42 days.

In the OVX RAL group, OPG was detected in the extracellular matrix, connective tissue, and discretely in the forming trabecular bone. OPG was moderately expressed at 14 days (Fig. 8), but the intensity increased at 42 days.
Fig. 2 Graph of blood clot percentage in OVX Ale, OVX NT, OVX Ral, and SHAM groups in at 14- and 42-day periods. Note statistically significant difference (*) in the comparisons between OVX RAL vs OVX NT at 14 days $(P<0.05)$ and OVX RAL vs SHAM at 42 days $(P<0.05)$

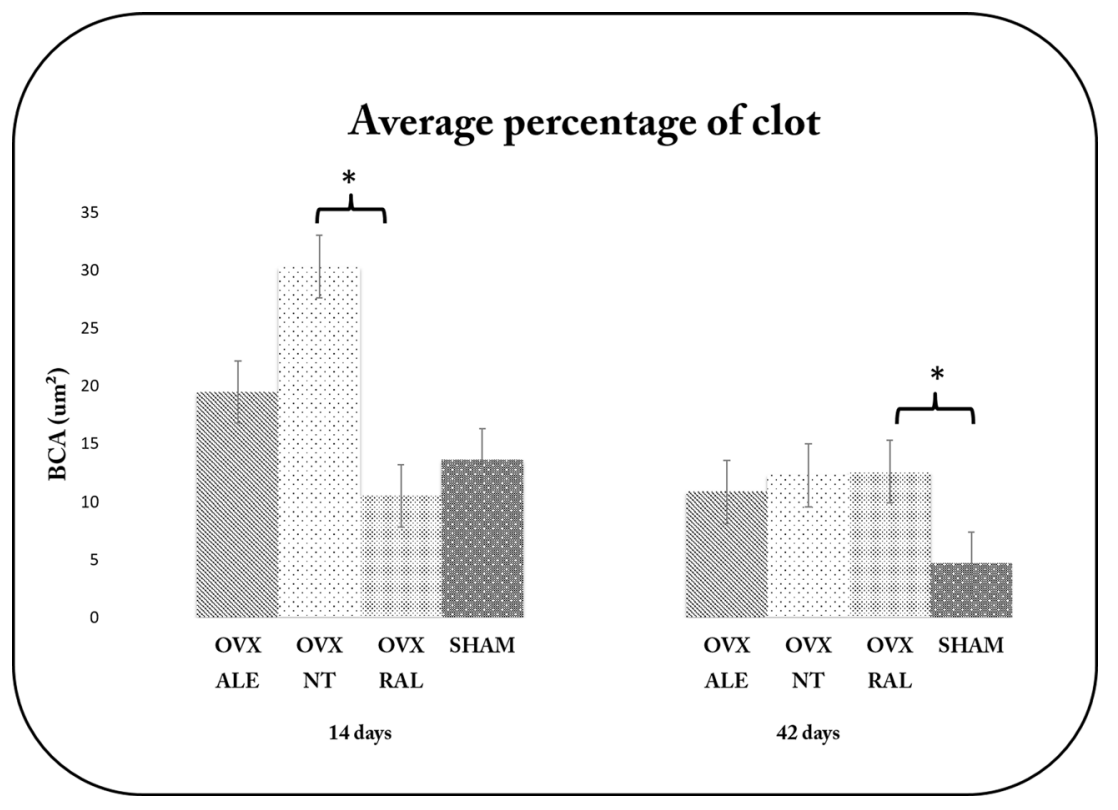


Fig. 3 Graph of connective tissue percentage in OVX Ale, OVX NT, OVX Ral, and SHAM groups in at 14- and 42-day periods. Note statistically significant difference (*) in the comparisons between OVX RAL VS OVX ALE and SHAM vs OVX ALE at 14 days $(P<0.05)$. There was no statistically difference between groups at 42 days $(P<0.05)$

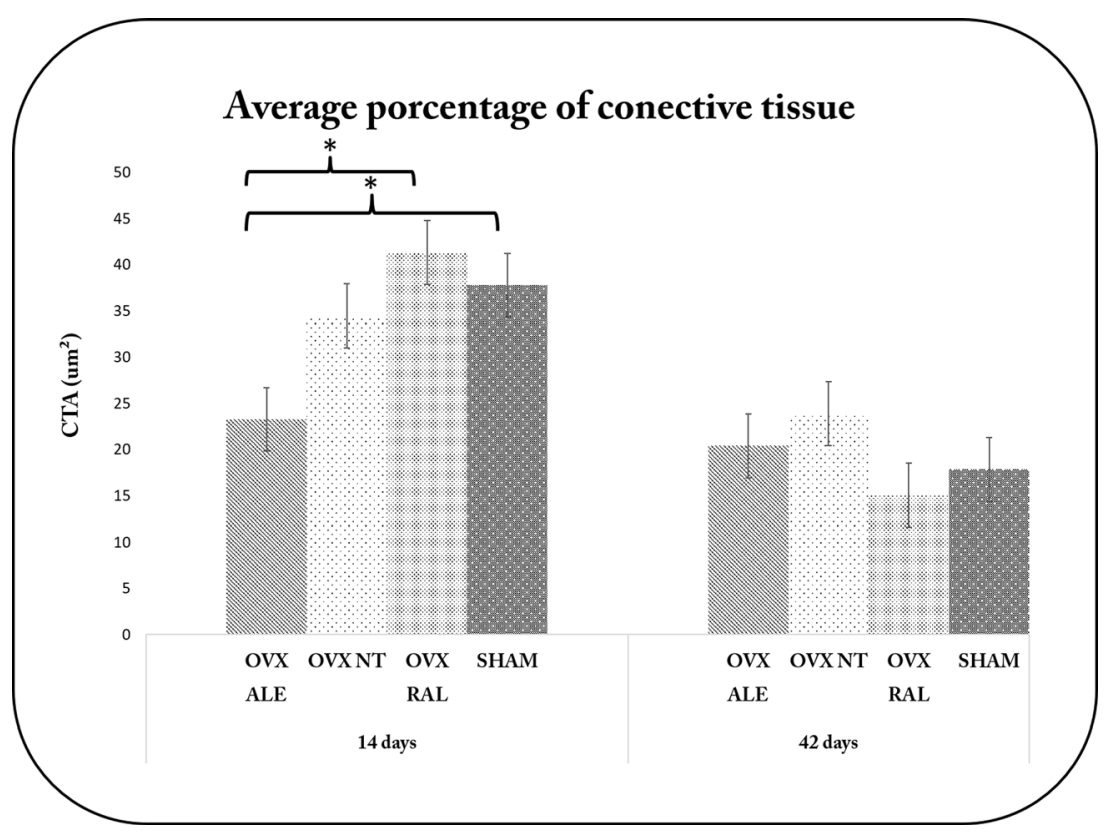

14 days and moderately to intensely expressed at 42 days (Fig. 7).

In the OVX RAL groups, RANKL was expressed by osteoblasts and specially by osteocytes. The bone trabeculae also exhibited RANKL-positive cells. RANKL was intensely expressed at 14 days and moderately expressed at 42 days (Fig. 8).

\section{Osteocalcin labeling}

In the SHAM group, a few osteocytes, the extracellular matrix, and the bone trabeculae stained positively for osteocalcin. The bone tissue was in a mature state, as noted by positive osteocyte labeling as well as osteocalcin precipitation in the
Fig. 4 Graph of bone formation percentage in OVX Ale, OVX NT, OVX Ral, and SHAM groups in the experimental periods of 14 and 42 days. There was no statistically significant difference between groups

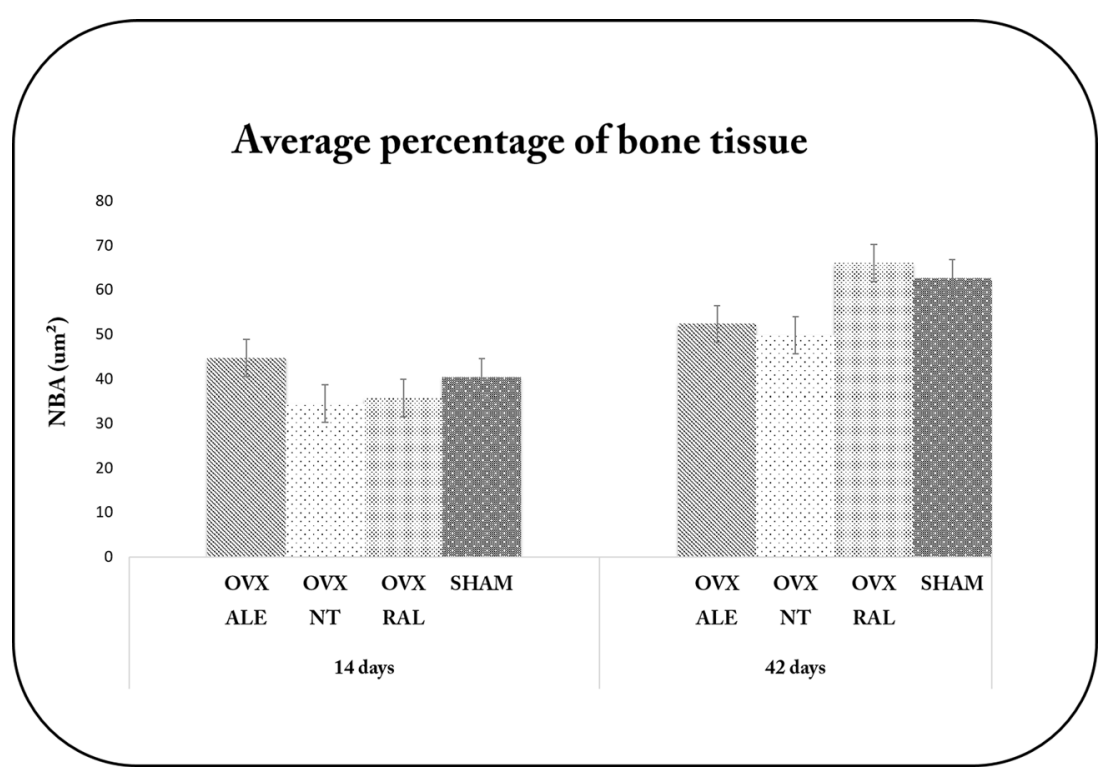




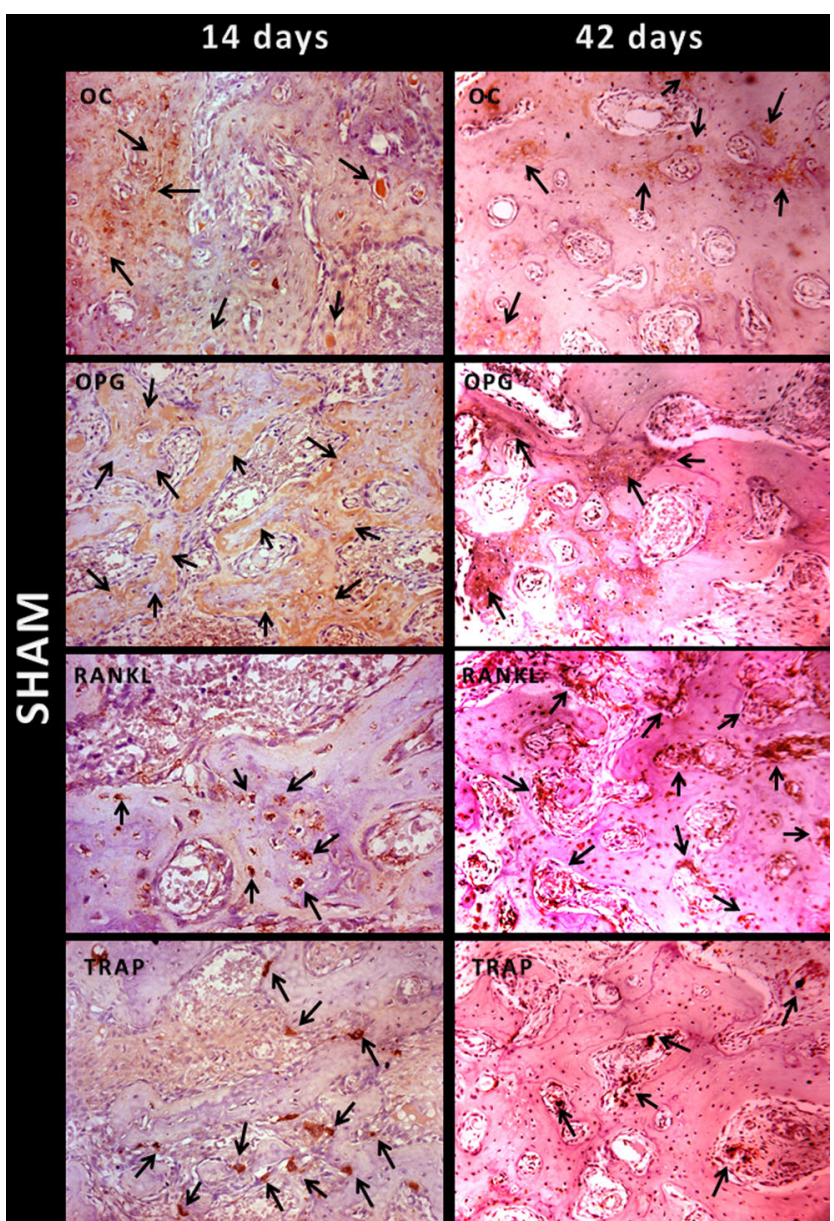

Fig. 5 Immunolabeling at 14 and 42 days after right upper incisive extraction of SHAM rats (OC, OPG, RANKL, and TRAP). Arrows indicate amount of positive labeling (original, $\times 200$ )

forming bone trabeculae. Osteocalcin was moderately to intensely expressed at 14 days, and moderately expressed at 42 days (Fig. 5).

In the OVX NT group, osteocalcin was strongly expressed by osteoblasts. The extracellular matrix also stained positively for osteocalcin. Some osteocytes presented discrete osteocalcin expression. The pattern of osteocalcin expression indicated that the bone tissue was immature compared to SHAM group. Osteocalcin expression was intense at 14 days and light to moderate at 42 days (Fig. 6).

In the OVX ALE group, osteocalcin was expressed by osteocytes and osteoblasts. Some extracellular matrix areas also expressed osteocalcin (Fig. 7). It is important to note that osteocalcin-positive osteoblasts and osteocytes indicated higher bone tissue maturity than in the other groups at 14 days; at 42 days, osteocalcin expression was light to moderate.

In the OVX RAL group, osteocalcin was moderately expressed by osteoblasts, osteocytes, and the extracellular matrix. Osteocalcin was moderately expressed at 14 days and moderately to intensely expressed at 42 days (Fig. 8). In the OVX RAL group, as in the OVX ALE group, the cellular

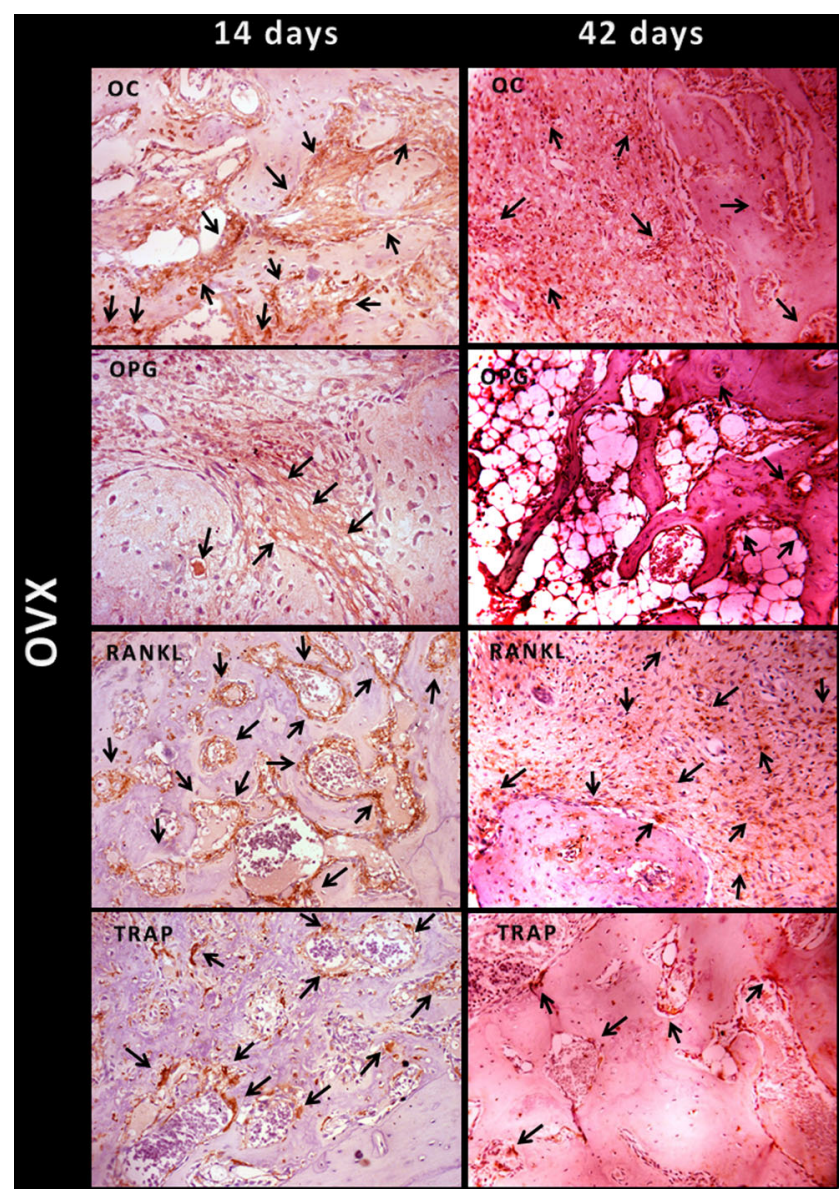

Fig. 6 Immunolabeling at 14 and 42 days after right upper incisive extraction of OVX NT rats (OC, OPG, RANKL, and TRAP). Arrows indicate amount of positive labeling (original, $\times 200$ )

responses indicated a lesser maturity level compared to the SHAM group. However, it is important to note that the maturity level was superior to that of the OVX NT group.

\section{TRAP labeling}

TRAP enzyme represents the osteoclast activity that occurs in forming bone tissue during bone multicellular units BMU activation. In the SHAM group, TRAP was moderately expressed by osteoclasts at 14 days, but only lightly expressed at 42 days (Fig. 5). In the OVX NT group, TRAP was intensely expressed by osteoclasts at 14 days, but only lightly expressed at 42 days (Fig. 6). In the OVX ALE or OVX RAL groups, TRAP was moderately expressed by osteoclasts at 14 and 42 days (Figs. 7 and 8 ).

\section{Discussion}

The percentage of bone formation was greater in the alendronatetreated group than in the raloxifene-treated group at 14 days. At 42 days, the results were inverted, i.e., the percentage of bone 
Fig. 7 Immunolabeling at 14 days after right upper incisive extraction of OVX ALE rats (OC, OPG, RANKL, and TRAP). Arrows indicate amount of positive labeling (original, $\times 200$ )

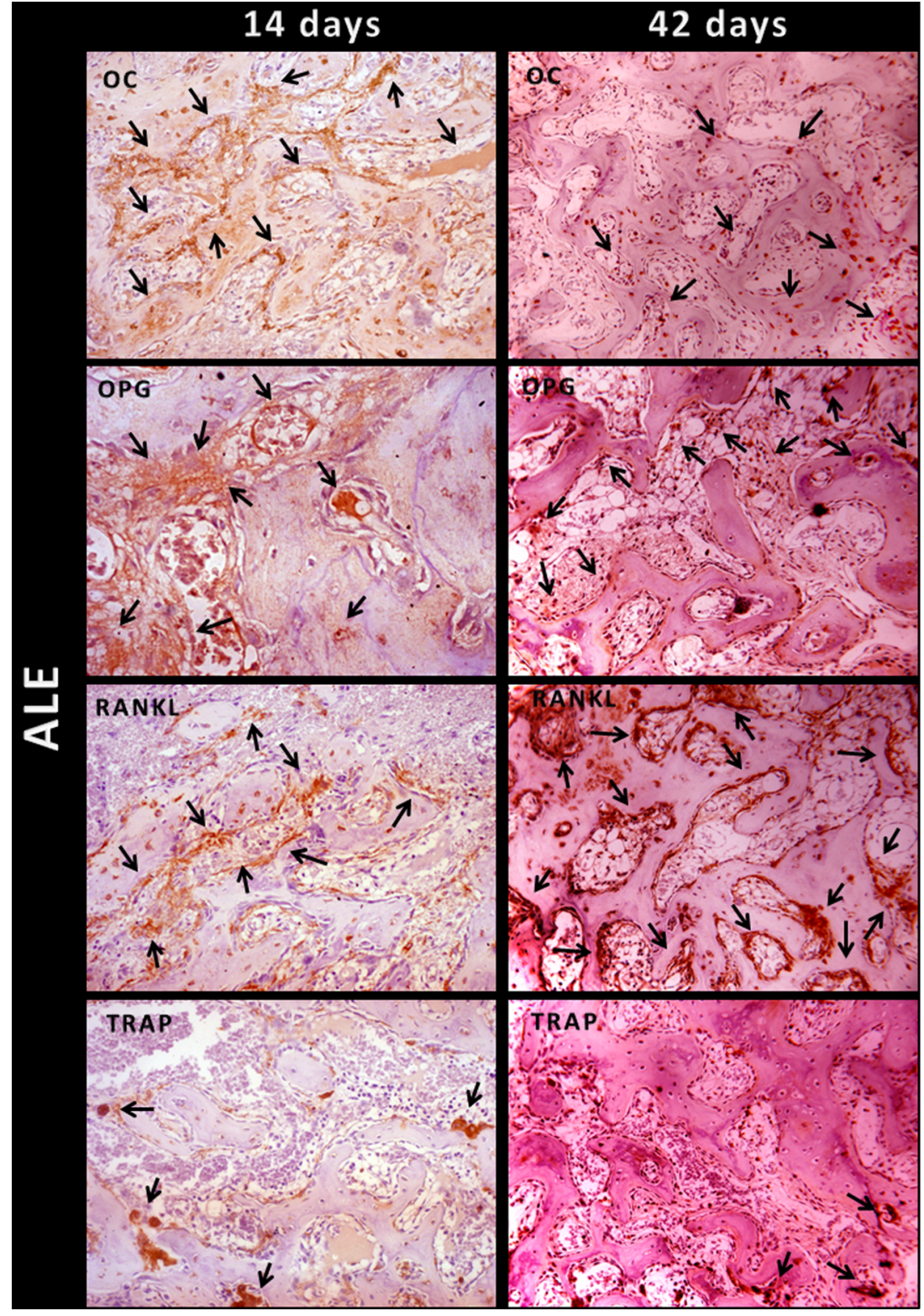

formation was greater in the raloxifene-treated group than in the alendronate-treated group. However, this bone tissue behavior was not statistically significant.

In this study, the alveolar healing process steps occurred according to what has been described in literature [21, 22] The experimental model successfully induced osteoporosis. However, in the treated groups (OVX ALE and OVX RAL), there was only a partial improvement of the alveolar healing process compared to OVX NT rats. Teófilo et al. [3] demonstrated that ovariectomy combined with a low-calcium diet induced osteoporosis to a greater extent than ovariectomy alone.

The alveolar healing process can be divided into three major steps: the exudative step, characterized by fibrin formation; the proliferative step, characterized by cell proliferation that will produce granulation tissue; and the repair step, characterized by collagen synthesis and ossification [23]. In this study, the three steps were evaluated through the quantification of blood clot, connective tissue, and bone tissue. Our aim was to evaluate the dynamics of the alveolar healing process in an osteoporotic situation and in the presence of drugs such as alendronate and raloxifene.

The SHAM group, which was considered the control group, showed the greatest responses regarding bone formation, as well as a decrease in blood clot and connective tissue area, indicating that the alveolar healing process occurred as expected [13]. The installed osteoporosis showed a greater decrease in bone formation response, represented by the lower percentage of bone formation at 14 and 42 days in the OVX NT group. The OVX NT group also presented the greatest 


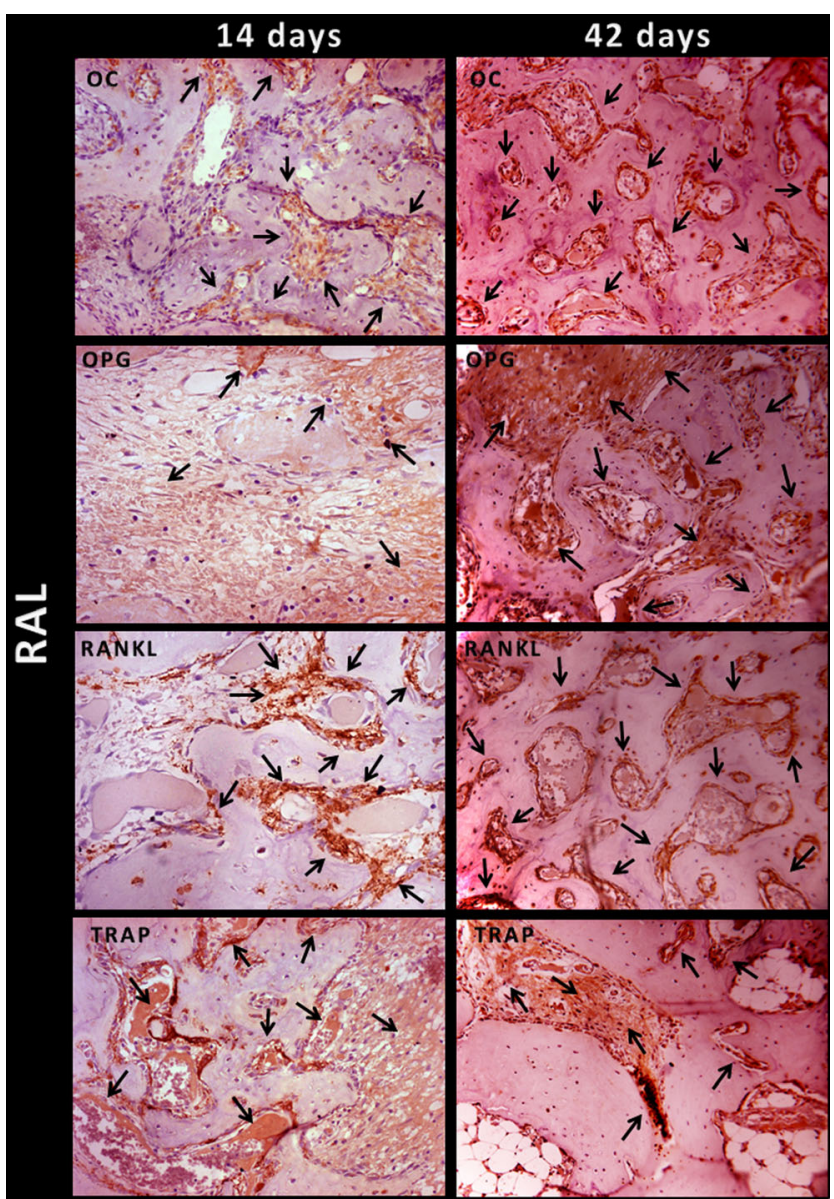

Fig. 8 Immunolabeling at 14 days after right upper incisive extraction of OVX RAL rats (OC, OPG, RANKL, and TRAP). Arrows indicate amount of positive labeling (original, $\times 200$ )

blood clot and connective tissue area at 42 days. Alendronate or raloxifene administration to rats with installed osteoporosis improved the bone formation response as expected. Initially, alendronate promoted greater responses than raloxifene as observed in the 14 days following the surgery. Otherwise, at 42 days, it was possible to observe that the OVX RAL and SHAM groups tended to present a greater bone formation response than the OVX ALE group. Therefore, raloxifene seemed to promote bone tissue responses that result in greater bone tissue dynamics. This is in agreement with the results obtained by Luvizuto et al. [20], who observed that raloxifene initially produces a less pronounced bone formation response. Otherwise, at 42 days, raloxifene promoted constant responses regarding the percentage of bone formation.

Considering the biological responses and cell signaling pathways at 14 days after tooth extraction, the period of greater metabolic activity during the alveolar healing process [13], it was possible to observe that osteoblast cells produced proteins to reach bone tissue equilibrium. The expressions of OPG and RANKL were similar across groups. Nevertheless, the expression of RANKL in osteoblasts was higher in the OVX NT group, suggesting preosteoclast activation. This finding is confirmed by studies that showed that high RANKL expression results in greater osteoclast activation [20, 24-26]. In the OVX RAL group, RANKL expression was slightly lower than that in other groups. Similar findings were obtained by Luvizuto et al. [24], who reported that raloxifene downregulated RANKL expression and TRAP activity.

Osteocalcin in bone tissue revealed differences in the quality of the new bone during the repair process. The SHAM group presented the highest osteocalcin expression and the OVX NT group presented the lowest osteocalcin expression. At 14 days, the expression of osteocalcin was higher in the OVX ALE group than in the OVX RAL group. These results justify the finding that, at 14 days after the surgery, the percentage of bone formation was lower in the OVX RAL group than in the OVX ALE group. These results corroborate with the work of Luvizuto et al. [20], who found that osteocalcin expression at 14 days after tooth extraction was higher in the SHAM group but lower in the OVX NT and OVX RAL groups. As mentioned above, raloxifene appear not to induce pronounced responses in the initial periods of administration.

The cytoarchitecture of alveolar bone healing on histological and immunohistochemical analyses showed that antiosteoporosis therapy with alendronate improved repair compared to controls, yet the response did not reach the results obtained in healthy rats or in osteoporotic rats treated with raloxifene. These findings were confirmed by RamalhoFerreira et al. [27], who evaluated alveolar bone turnover in osteoporotic rats by confocal laser microscopy; using a technique in which calcium precipitation is stained with fluorochromes (calcein and alizarin red), the authors showed that osteoporotic rats and osteoporotic rats treated with alendronate exhibited higher levels of calcein and lower levels of alizarin red, representing higher amount old bone and, and lower amount of new bone, respectively. Thus, alendronate acts as a potent antiresorptive medication despite no bone turnover or an increase in new bone.

Clinically, tooth extraction procedures, scaling and root planning, or even periodontitis may lead to contamination and exposure of alveolar bone in patients with osteoporosis treated with alendronate. If bone turnover is compromised (i.e., there is no cell renewal), there is increased predisposition to develop medication related osteonecrosis of the jaw MRONJ, a condition of difficult surgical resolution [28].

There is no question that in the treatment of osteoporosis for prevention of vertebral fractures and long bone, alendronate is effective, as proven by randomized clinical trials [29-31]. However, in relation to the oral cavity, in addition to contamination, there occur microtraumas induced by masticatory load. In these patients, the turnover will be compromised.

The immunohistochemical results at 42 days confirmed the tendency observed in the histometric results that the OVX RAL group was superior to the OVX ALE group. It was clear that there was an increase of proteins involved in higher bone 
formation and bone maturation in the rats treated with raloxifene (osteocalcin and OPG). On the other hand, there was a decrease in protein expression in the rats treated with alendronate. Ramalho-Ferreira et al. [27] evaluated the same experimental groups using fluorochrome precipitation in the alveolar bone, and also showed that alendronate presented higher amount of old bone and lower new bone (calcein/alizarin precipitation), thus corroborating with the results of the present study.

An interesting observation was the constant expression of TRAP in the OVX ALE group as opposed to RANKL, whose expression increased at 42 days. As observed in all metabolic reactions, this study showed higher RANKL signaling when alendronate induced antiresorptive responses. However, bisphosphonates promote inactivation and apoptosis of osteoclasts, leading to no active resorption.

During bone homeostasis, the rate of bone formation equals the rate of bone resorption. The predominance of one of these responses can produce harmful responses to the bone dynamics $[32,33]$.

The osteoblast is a key cell in the command of bone tissue responses, releasing factors that directly interfere with osteoclast response through paracrine signals. On the other hand, osteoclasts, through negative feedback responses, interfere with osteoblast activity. Therefore, one cell acts in function of the other. Hormones, as well as systemic and local factors may directly interfere with bone cell equilibrium [25].

An attempt to obtain pharmacological alternatives that could be used in osteoporosis treatment has led to the development of estrogen receptor modulators. Raloxifene belongs to this pharmacological class, and is the only approved by the FDA for clinical use [11]. Raloxifene treatment and hormone replacement affects the local production of cytokines and growth factors, decreases osteoclast formation, increases osteoblast and osteocyte life span, and exerts an antioxidant effect by inhibiting ovariectomy-induced osteocyte apoptosis [34, 35]. Therefore, raloxifene acts by inhibiting bone resorption as well as by stimulating cells that produce bone matrix. For this reason, it is possible that raloxifene has better long-term response than alendronate.

In this study, pharmacological treatment improved the bone quality compared to OVX NT. However, the bone quality was inferior to that of the SHAM group. The quality of osseointegration in bone tissue with the same observed characteristics, with thinner bone trabeculae, and with decreased expression of the studied proteins, is still unknown. Therefore, new studies evaluating osseointegration must be performed.

\section{Conclusions}

Within the limitations of our study, it was possible to conclude the following:
- Ovariectomy combined with a low-calcium diet served as an experimental model for osteoporotic bone loss.

- Alveolar healing process dynamics were negatively affected in osteoporotic rats.

- Alendronate or raloxifene administration improved alveolar healing process dynamics.

Acknowledgments The authors would like to express their gratitude to FAPESP (2010/04366-1) for the scholarship provided to the first author (Ramalho-Ferreira, G.) and FUNDUNESP (01117911 - DFP) for the grant support.

Funding This study was partly supported by Research support foundation from São Paulo State (No. 2010/04366-1).

\section{Compliance with ethical standards}

Conflict of interest The authors declare that they have no conflict of interest.

Ethical approval The procedures and protocol design described here were approved by the Ethics Committee in Animal Experimentation of Aracatuba Dental School, UNESP-Univ Estadual Paulista, Brazil (approval \#2010/003045).

Informed consent For this type of study, formal consent is not required.

\section{References}

1. Kribbs PJ (1990) Comparison of mandibular bone in normal and osteoporotic women. J Prosthet Dent 63:218-222

2. Tyagi AM, Srivastava K, Mansoori MN, Trivedi R, Chattopadhyay N, Singh D (2012) Estrogen deficiency induces the differentiation of IL-17 secreting Th17 cells: a new candidate in the pathogenesis of osteoporosis. PLoS One 7(9):e44552

3. Teófilo JM, Azevedo AC, Petenusci SO, Mazaro R, LamanoCarvalho TL (2003) Comparison between two experimental protocols to promote osteoporosis in the maxilla and proximal tibia of female rats. Pesq Odontol Bras 17(4):302-306

4. Teófilo JM, Brentegani LG, Lamano-Carvalho TL (2004) Bone healing in osteoporotic female rats following intra-alveolar grafting of bioactive glass. Arch Oral Biol 49(9):755-762

5. Manolagas SC, Jilka RL (1995) Bone marrow, cytokines, and bone remodeling. Emerging insights into the pathophysiology of osteoporosis. N Engl J Med 332(5):305-311

6. Zavras AI. The impact of bisphosphonates on oral health: lessons from the past and opportunities for the future. Ann N Y Acad Sci (2011) 1218.1 55-61.

7. Marx RE (2003) Pamidronate (Aredia) and zoledronate (Zometa) induced avascular necrosis of the jaws: a growing epidemic. J Oral Maxillofac Surg 61(9):1115-1117

8. Giro G. (2006) Avaliação radiográfica e biomecânica da influência da osteoporose induzida em ratas e seu tratamento com alendronato e estrógeno, sobre tecido ósseo ao redor de implantes com osseontegração estabelecida. Tese de Mestrado apresentada para obtenção do título de Mestre em Periodontia pela Faculdade de Odontologia de Araraquara- UNESP

9. Russel RG, Rogers MJ (1999) Biphosphonates: from the laboratory to the clinic and back again. Bone 25:97-106 
10. Iwamoto J, Yeh JK, Schmidt A, Rowley E, Stanfield L, Takeda T, Sato M (2005) Raloxifene and vitamin K2 combine to improve the femoral neck strength of ovariectomized rats. Calcif Tissue Int 77(2):119-126

11. Ettinger B, Black DM, Mitlak BH, Knicherbocker RK, Nickelsen T, Genant HK, et al. (1999) Reduction of vertebral fracture risk in postmenopausal women with osteoporosis treated with raloxifene: results from a 3-year randomized clinical trial. Multiple Outcomes of Raloxifene Evaluation (MORE) investigators. JAMA 282:637645

12. Barrett-Connor E, Grady D, Sashegyi A, Anderson PW, Cox DA, Hoszowski K, et al. (2002) Raloxifene and cardiovascular events in osteoporotic postmenopausal women: four-year results from the MORE (Multiple Outcomes of Raloxifene Evaluation) randomized trial. JAMA 287:847-857

13. Okamoto T, Russo MC (1973) Wound healing following tooth extraction: histochemical study in rats. Rev Fac Odontol Araçatuba 2: 153-169

14. Carvalho ACP, Okamoto T (1987) Cirurgia bucal: fundamentos experimentais aplicados a clínica. Panamericana, São Paulo

15. Von Wowern N, Kollerup G (1992) Symptomatic osteoporosis: a risk factor for residual ridge reduction of the jaws. J Prosthet Dent 67:656-660

16. Elsubeihi ES, Heersch JMN. Effects of postmenopausal osteoporosis in the mandible. In: Zarb G, Lekholm U, Albrektsson T, Tenenbaum H. Aging, osteoporosis and dental implants. London: Quintessence Books, 2002: 207-215.

17. Ramalho-Ferreira G et al. (2015) Raloxifene enhances peri-implant bone healing in osteoporotic rats. Int J Oral Maxillofac Surg 44(6): 798-805

18. Long JA, Evans HM (1922) The oestrous cycle in the rat and its associated phenomena. Mem Univ Calif 6:1-148

19. Da Paz LH, de Falco V, Teng NC, Dos Reis LM, Pereira RM, Jorgett V (2001) Effect of 17 beta-estradiol or alendronate on the bone densitometry, bone histomorphometry and bone metabolism of ovariectomized rats. Braz J Med Res 34(8):1015-1022

20. Luvizuto ER, Dias SM, Queiroz TP, Okamoto T, IR G Jr, Okamoto R, Dornelles RC (2010) Osteocalcin immunolabeling during the alveolar healing process in ovariectomized rats treated with estrogen or raloxifene. Bone 46(4):1021-1029

21. Shyng YC, Devlin H, Riccardi D, Sloan P (1999) Expression of cartilage-derived retinoic acid-sensitive protein during healing of the rat tooth-extraction socket. Arch Oral Biol 44(9):751-757

22. de Oliveira PT, Zalzal SF, Irie K, Nanci AJ (2003) Early expression of bone matrix proteins in osteogenic cell cultures. Histochem Cytochem 51(5):633-641

23. Amler MH (1969) The time sequence of tissue regeneration in human extraction wounds. Oral Surg Oral Med Oral Pathol 27:309-318
24. Luvizuto ER, Queiroz TP, Dias SM, Okamoto T, Dornelles RC, IR G Jr, Okamoto R (2010) Histomorphometric analysis and immunolocalization of RANKL and OPG during the alveolar healing process in female ovariectomized rats treated with estrogen or raloxifene. Arch Oral Biol 55(1):52-59

25. Giner M, Rios MJ, Montoya MJ, Vázquez MA, Miranda C, PérezCano R (2011) Alendronate and raloxifene affect the osteoprotegerin/RANKL system in human osteoblast primary cultures from patients with osteoporosis and osteoarthritis. Eur $\mathbf{J}$ Pharmacol 650(2-3):682-687

26. Ostrowska Z, Ziora K, Oświęcimska J, Swiętochowska E, Szapska B, Wołkowska-Pokrywa K, Dyduch A (2012) RANKL/RANK/ OPG system and bone status in females with anorexia nervosa. Bone 50(1):156-160

27. Ramalho-Ferreira $\mathrm{G}$ et al. (2015) Alveolar bone dynamics in osteoporotic rats treated with raloxifene or alendronate: confocal microscopy analysis. J Biomed Opt 20 3(3):038003-038003

28. Ruggiero SL, Dodson TB (2014) American Association of Oral and Maxillofacial Surgeons position paper on medication-related osteonecrosis of the jaws-2014 update. J Oral Maxillofac Surg 72(12):2381-2382

29. Murad MH, Drake MT, Mullan RJ, Mauck KF, Stuart LM, Lane MA, Li T (2012) Comparative effectiveness of drug treatments to prevent fragility fractures: a systematic review and network metaanalysis. J Clin Endocrinol Metab 97(6):1871-1880

30. Bauer DC, Garnero P, Hochberg MC, Santora A, Delmas P, Ewing SK, Black DM (2006) Pretreatment levels of bone turnover and the antifracture efficacy of alendronate: the fracture intervention trial. $\mathrm{J}$ Bone Miner Res 21(2):292-299

31. Schwartz AV, Bauer DC, Cummings SR, Cauley JA, Ensrud KE, Palermo L, Black DM (2010) Efficacy of continued alendronate for fractures in women with and without prevalent vertebral fracture: the FLEX trial. J Bone Miner Res 25(5):976-982

32. Simonet WS, Lacey DL, Dunstan CR, Kelley M, Chang MS, Luthy R, et al. (1997) Osteoprotegerin: a novel secreted protein involved in the regulation of bone density. Cell 89:309-319

33. Bucay N, Sarosi I, Dunstan CR, Morony S, Tarpley J, Capparelli C, Scully S, Tan HL, Xu W, Lacey DL, Boyle WJ, Simonet WS (1998) Osteoprotegerin-deficient mice develop early onset osteoporosis and arterial calcification. Genes Dev 12(9):1260-1268

34. Cao Y, Mori S, Mashiba T, Westmore MS, Ma L, Sato M, et al. (2002) Raloxifene, estrogen and alendronate affect the processes of fracture repair differently in ovariectomized rats. J Bone Miner Res 17:2237-2246

35. Stepan JJ, Alenfeld F, Boivin G, Feyen JH, Lakatos P (2003) Mechanisms of action of antiresorptive therapies of postmenopausal osteoporosis. Endocr Regul 37:227-240 